\title{
Tetracoordinated quasicrystals
}

\author{
J. Peters and H.-R. Trebin \\ Institut für Theoretische und Angewandte Physik der Universität Stuttgart, \\ Pfaffenwaldring 57, D-7000 Stuttgart 80, Federal Republic of Germany \\ (Received 19 March 1990)
}

\begin{abstract}
Current model networks for amorphous Ge contain five-membered rings and pentagonal dodecahedra to explain why in the radial distribution function the third peak of the diamond structure is missing. By presenting an algorithm based on a decoration of the three-dimensional Penrose quasilattice, we prove that this local pentagonal symmetry can be extended globally to an icosahedral quasicrystalline tetracoordinated network. Its structural elements and topological properties coincide with previous hand-built models of random networks. Thus it is suitable for simulating bulk properties of amorphous semiconductors.
\end{abstract}

The radial distribution function (RDF) of $a$-Si and $a$ $\mathrm{Ge}$, derived from the ring-shaped diffraction patterns, indicates that the basic tetrahedral coordination of crystalline $\mathrm{Si}$ and $\mathrm{Ge}$ is preserved with bond-length variations of about $3 \%$ to $6 \%$ (Refs. 1 and 2 ) and bond-angle distortions of $5 \%$ to $10 \% .^{3,1}$ The low intensity of the third RDF peak as compared to the crystalline RDF, however, indicates fundamental modifications in the dihedral-angle distribution, which are not yet clearly elucidated. ${ }^{4,5}$ The dihedral angle measures the relative rotation of neighboring tetrahedral arrangements about their common bond. In the diamond structure, all dihedral angles are $60^{\circ}$. In the continuous-random-network (CRN) models for amorphous semiconductors of Polk ${ }^{6}$ and of Connell and Tem$\mathrm{kin}^{4}$ the dihedral angle varies continuously from $0^{\circ}$ (denoted "eclipsed") to a modest maximum at $60^{\circ}$ (denoted "staggered").

Exclusive use of the eclipsed configuration leads to planar five-membered rings and to clusters in the form of pentagonal dodecahedra, Fig. 1(a). Earlier models of Coleman and Thomas ${ }^{7}$ and of Grigorovici and Mănăilă ${ }^{2,8}$ used mixtures of the diamond structure and of pentagonal dodecahedra to explain the missing peak and thus admitted only staggered and eclipsed configurations. However, no rule was provided on how to interconnect the

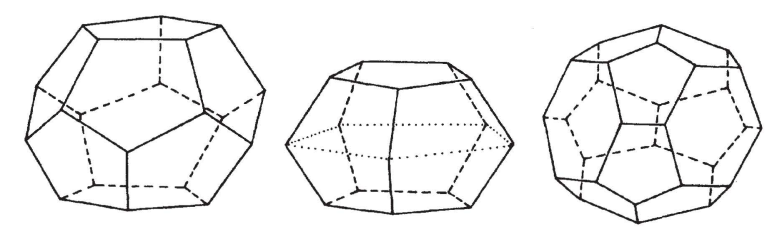

FIG. 1. With staggered and eclipsed bonds, a planar fivemembered ring can only be continued to form a pentagonal dodecahedron (a) or the so-called "barrelan" (b); dots indicate the boat-type six-membered rings' creases and do not represent bonds. The "hexadecahedron" (c) includes planar hexagons and distorted tetrahedral arrangements. two structural elements.

In 1980, Dandoloff, Döhler, and Bilz ${ }^{9}$ investigated the low-lying TA-phonon branches of crystalline $\mathrm{Si}$ and $\mathrm{Ge}$, which contain only "seat-type" six-membered rings. A softening of the TA mode at the $L$ point would transform the seats into more flexible "boat-type" six-membered rings. With the boat-type rings and with planar fivemembered rings they developed a new elementary subunit in addition to the dodecahedron: a barrel-shaped 15atom cluster called "barrelan," Fig. 1(b). Stacking both subunits and thus using only staggered and eclipsed bonds, they obtained a finite macrocluster of exact dodecahedral symmetry. However, a global packing of dodecahedra and barrelans could not be achieved.

All these models were hand built and limited to less than 500 atoms. The RDF's fit quite well-though not perfectly ${ }^{4,7,8}$ - with experimental data. The major problem was the lack of any systematic construction rules, which would allow one to create nearly perfect tetrahedrally connected networks of arbitrary extension.

One characteristic feature of the models, the incorporation of planar five-membered rings, is indeed supported by the observation of pentagonal structures in multiply twinned $\mathrm{Ge},{ }^{10-12}$ and in the crystalline Ge-III (ST-12) structure. ${ }^{13}$ The pentagonal dodecahedron and icosahedron are dual and possess the same symmetry. If dodecahedra are building blocks of amorphous networks, the question poses itself, whether the local symmetry can propagate to a global one.

First, systematic principles came from the "crystallography of the amorphous state" in metallic glasses by Kléman and Sadoc, ${ }^{14}$ Sethna, ${ }^{15,16}$ and Nelson. ${ }^{17}$ In amorphous metals the dominating substructures are assumed to be icosahedra, the locally densest packing of 13 atoms. This packing cannot be continued in flat Euclidean space, but can in the form of the polytope $\{3,3,5\}$ on the positively curved hypersphere $S^{3}$ embedded in fourdimensional space. By dualizing this polytope, i.e., replacing each of its 600 tetrahedral cells by a vertex, the tetracoordinated polytope $\{5,3,3\}$ was constructed, ${ }^{5}$ which consists of 120 dodecahedra. Substitution of all 
vertices in the polytope $\{5,3,3\}$ by centered tetrahedra results in a polytope of 3000 vertices, which is a packing of dodecahedra and barrelans and which is locally identical to the hand-built model of Dandoloff et al. ${ }^{9}$

Decurving procedures ${ }^{5,17,18}$ were developed, but no systematic algorithms were presented for space-filling structures. Again, help came from the theory of metallic glasses, which share the problem of decurving $S^{3}$ and of the space-filling continuation of fivefold symmetric clusters. For the structure of metallic alloys, which display diffraction patterns of icosahedral symmetry, ${ }^{19}$ icosahedral quasilattices were proposed. These "threedimensional Penrose tilings" tesselate space with two types of rhombohedral cells in a quasiperiodic way. ${ }^{20}$ Can, for networks, the local fivefold symmetry be so dominant as to give rise to quasicrystalline semiconductors? Tetracoordinated quasicrystals were constructed by dualizing the "truncated-icosahedra" decoration of the Penrose quasilattice, which divides three-dimensional space into distorted tetrahedra. ${ }^{21-23}$ However, these networks include four-membered rings which excessively distort the bond angles and hence should not be accepted as reasonable structural elements. Further attempts were made by Ishii ${ }^{18}$ and Olami and Alexander, ${ }^{24}$ but the resulting structures contain an abundant amount of dangling bonds.

Summarizing, it seems that up to now no systematic algorithms have been available which allow construction of infinite networks that are nearly perfectly tetrahedrally coordinated, nonperiodic, and almost isotropic with regard to bond directions and local cluster orientations.

Here, we present an almost perfect tetrahedrally coordinated network $(0.78 \%$ of dangling bonds) based on a symmetric decoration of the two Penrose rhombohedra (Fig. 2). These tesselate the whole three-dimensional space in the form of a Penrose quasilattice. The resulting tetracoordinated quasicrystals are systematically constructable and hence not random. But they can be regarded as an excellent approximation of the amorphous state because (i) they are nonperiodic, (ii) the icosahedrally symmetric distribution of their bond-directions is almost isotropic, ${ }^{25}$ and (iii) the local structure coincides with that of current models of the disordered state.

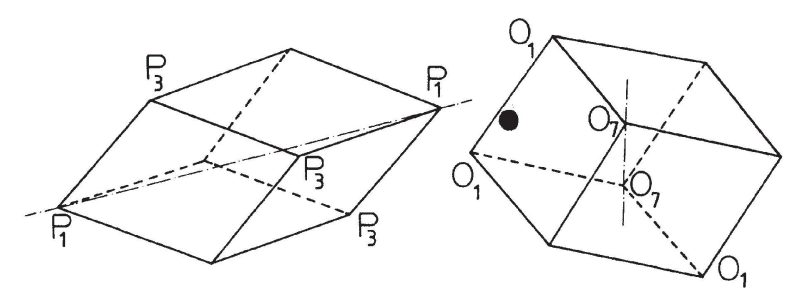

FIG. 2. Penrose tiles: a prolate and an oblate rhombohedron spanned by icosahedrally oriented edges. The four types of corners are designated according to their internal solid angles (in units of $\pi / 5$ ) as $P_{1}, P_{3}, O_{1}$, and $O_{7}$. The dash-dotted lines represent threefold axes.
From metallic quasicrystals we know that many of their macroscopic properties resemble much more those of their amorphous modifications than of their crystalline ones. $^{26}$

One model unites several previously proposed ideas. We restrict ourselves to staggered and eclipsed bonds, thereby limiting the set of elementary structures. Exclusive use of either staggered or eclipsed bonds leads to the seat-type six-membered ring or the planar fivemembered ring, respectively, whereas the boat-type sixmembered rings are formed wherever both bond types meet. Five-membered rings necessarily lead to the structural subunits dodecahedron and barrelan (Fig. 1). Our model's local order is very close to that of Dandoloff et al. ${ }^{9}$ We derived a systematic modification of their local dodecahedron-barrelan packing towards an infinite structure by using the "decorated-quasilattice" concept.

An essential demand is a symmetric decoration of the two Penrose rhombohedra, such that they can be stacked without matching rules or a subsequent removal of atoms. The neighborhood of a Penrose quasilattice vertex is classified by the types of its surrounding rhombohedral corners, which are denoted $P_{1}, P_{3}, O_{1}$, and $O_{7}$ (Fig. 2). Inspection of the possible vertex and edge neighborhoods forbids the positioning of atoms on the rhombohedral corners or edges. ${ }^{27}$ Symmetric decoration therefore allows atoms to be placed only inside the rhombohedra or on their faces.

Using the common icosahedral symmetry of both the dodecahedron and all the quasilattice edge directions, we center a dodecahedron on each Penrose vertex with its pentagonal faces oriented normal to the edges of the rhombohedral tiles. Since all these dodecahedra surrounding the vertices are aligned, their mutually facing pentagons stand in a "staggered" position. To achieve face matching, an additional $(\pi / 5)$-rotated dodecahedron must be inserted along the rhombohedral edges. But simple stacks of three successive dodecahedra cause overlaps and wrong coordinations of two dodecahedra along the short diagonal of the oblate rhombohedron. ${ }^{28}$ We resolved these problems by including the central structure of the Dandoloff-Döhler-Bilz model: barrelans are inserted between the dodecahedra, perfectly fitting along the rhombohedral edges and with minor distortions between mid-edge dodecahedra (Fig. 3).

Continuation of the tetrahedral network into the prolate rhombohedron (PR) is straightforward: the 24 hatched pentagons (Fig. 3) inside the PR are capped with barrelans. These group with minor cracks around two "hexadecahedra," Fig. 1(c), each bounded by 12 pentagonal and 4 hexagonal faces. They are centered on the PR's threefold axis and face each other with a hexagonal ring. This pair of hexagons is automatically connected via the boat-type six-membered rings of some barrelans. (The inclusion of planar hexagons is encouraged by RDF measurements showing a small peak corresponding to their diagonal distance. ${ }^{29}$ )

It is more difficult to complete the tetrahedral network inside the oblate rhombohedron (OR). The dodecahedra surrounding the $\mathrm{O}_{7}$ corners in Fig. 2 provide a pair of atoms on the short OR-diagonal separated by 1.49 (in 


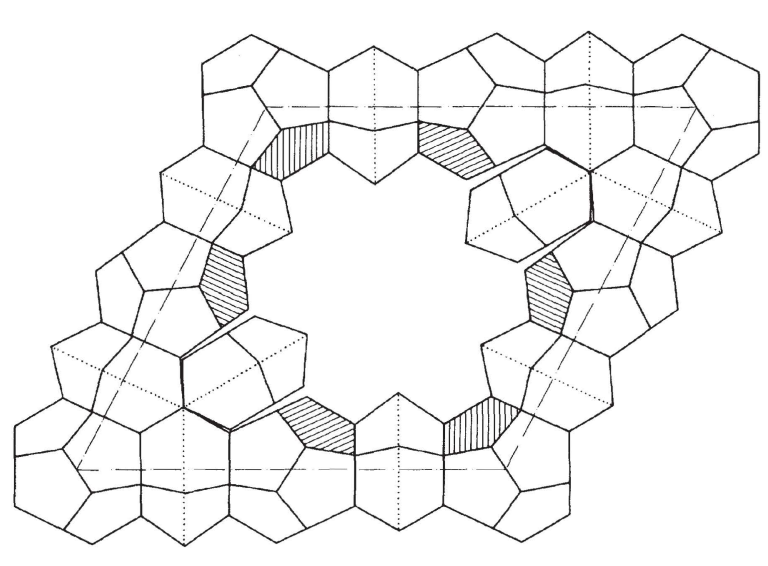

FIG. 3. Stacking of dodecahedra and barrelans along the rhombohedra's edges (dash-dotted line) and inserted barrelans between mid-edge dodecahedra; the crack angles are $5^{\circ}$.

units of the dodecahedral edge length). After a modest distortion of the two dodecahedra, these atoms are connected by a staggered bond. Of the six barrelans next to the $\mathrm{O}_{7}$ corners some atoms are not yet tetracoordinated inside the OR. They can be grouped to form six slightly distorted tetrahedra, which we center by additional atoms. Except for one atom of each of the six adjoining mid-edge dodecahedra, all OR-internal atoms now are tetracoordinated. As a consequence of several topological peculiarities of their neighborhood, these six atoms per OR must be left only threefold coordinated, each showing one dangling bond.

This decoration yields 170 atoms per prolate and 110

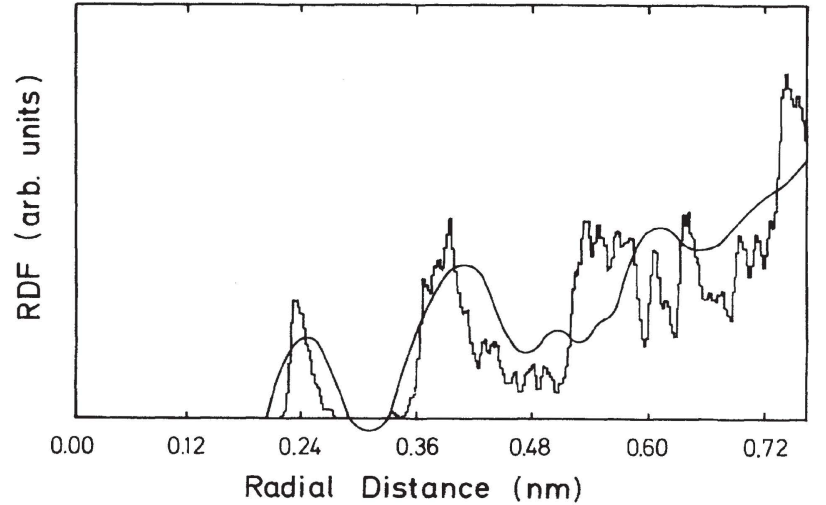

FIG. 4. Radial distribution function of the relaxed quasicrystalline network scaled to an average bond length of $0.24 \mathrm{~nm}$ for Ge and comparison with experimental values from Ref. 31.

per oblate rhombohedron, leaving six of the OR atoms with one dangling bond. Interesting connections to the close-packed $(\mathrm{A} 1, \mathrm{Zn})_{49} \mathrm{Mn}_{32}$ quasicrystal structure of Henley and Elser ${ }^{23}$ will be reported elsewhere.

When the decorated rhombohedra are stacked to an infinite Penrose quasilattice, one obtains the following statistical data: the ratio of five- to six-membered rings is $1: 2.062$, the ratio of staggered to eclipsed bonds is $1: 1.420$, and the dangling-bond density is $0.78 \%$.

The rational approximants ${ }^{30}$ of the decorated quasilattice are most suitable for simulations of physical properties with periodic boundary conditions and hence without surface effects. Even for the lowest indices $(1 / 0,1 / 1,2 / 1, \ldots)$ of the rational approximation, the numbers of $1120,4720,20000, \ldots$, atoms per cubic unit cell are very large. Thus a model network is available,

TABLE I. Comparison among the numbers of returning walks of $n$ steps in the diamond-lattice, two CRN models (selected starting atom) (Refs. 4 and 6), Sadoc and Mosseri's polytope "240" (taken from Ref. 5, p. 474), and in our 1/1 approximant (averaged for arbitrary starting atom).

\begin{tabular}{|c|c|c|c|c|c|}
\hline$n$ & $\begin{array}{c}\text { Diamond } \\
\text { lattice }\end{array}$ & $\begin{array}{l}\text { Connell's } \\
\text { CRN }^{\mathrm{a}}\end{array}$ & $\begin{array}{l}\text { Polytope } \\
\text { " } 240 "\end{array}$ & $\begin{array}{l}\text { Polk's } \\
\text { CRN }^{\mathrm{b}}\end{array}$ & $\begin{array}{l}\text { Present } \\
\text { model }\end{array}$ \\
\hline 1 & 0 & 0 & 0 & 0 & 0 \\
\hline 2 & 4 & 4 & 4 & 4 & 3.98 \\
\hline 3 & 0 & 0 & 0 & 0 & 0 \\
\hline 4 & 28 & 28 & 28 & 28 & 27.8 \\
\hline 5 & 0 & 0 & 0 & 4 & 4.88 \\
\hline 6 & 256 & 268 & 268 & 244 & 242.4 \\
\hline 7 & 0 & 0 & 0 & 90 & 102.5 \\
\hline 8 & 2716 & 2982 & 3004 & 2416 & 2406.9 \\
\hline 9 & 0 & 0 & 0 & 1564 & 1704.9 \\
\hline 10 & 31504 & 35895 & 36784 & 26016 & 25999.5 \\
\hline
\end{tabular}

${ }^{a}$ Reference 4 .

${ }^{\mathrm{b}}$ Reference 6 
constructed by a well-defined algorithm and adjustable in its size to physical requirements and to computer power. Randomness can be gradually introduced by applying the decoration to random Penrose tilings.

We have investigated the $1 / 1$ rational approximant with 4720 atoms and 72 dangling bonds per unit cell. Considering the cyclic boundaries, we relaxed the structure by minimizing the Keating energy, which rates all bond-length and bond-angle distortions. ${ }^{18}$ The structure proved quite stable apart from minor shifts of the atoms: During the relaxation the standard deviation of the bond-length distribution was reduced from $12 \%$ to $4 \%$, while that of the bond-angle distribution remained stable at $6.5 \%$, in good agreement with the experimental values of $3 \%$ to $6 \%$ and $5 \%$ to $10 \%$, respectively. The density of $96.5 \%$ as compared to the crystalline phase is in the range of experimental data. In the calculated RDF (Fig. 4) the third crystalline peak at $0.46 \mathrm{~nm}$ is missing, as expected. The typical dodecahedral shell at $0.55 \mathrm{~nm}$, however, is too pronounced compared with the RDF's shown in Refs. 4, 6, and 8. Finally, we compared the statistics of returning walks (reflecting the network topology) with several existing models and found a strong resemblance to Polk's CRN model (Table I). Thus, although our intention primarily has been to construct a tetracoordinated quasicrystal, we have found a structure which shares many properties with model networks for amorphous semiconductors.
${ }^{1} \mathrm{H}$. Overhof and P. Thomas, Electronic Transport in Hydrogenated Amorphous Semiconductors, Springer Tracts in Modern Physics (Springer, Berlin, in press).

${ }^{2}$ R. Grigorovici, Mat. Res. Bull. 3, 13 (1968).

${ }^{3}$ J. C. Knights, in The Physics of Hydrogenated Amorphous Silicon, Vol. 55 of Topics in Applied Physics, edited by J. D. Joannopoulos and G. Lucovsky (Springer-Verlag, Heidelberg, 1984).

${ }^{4}$ G. A. N. Connell and R. J. Temkin, Phys. Rev. B 9, 5323 (1974).

${ }^{5}$ J. F. Sadoc and R. Mosseri, Philos. Mag. B 45, 467 (1982).

${ }^{6}$ D. E. Polk, J. Non-Cryst. Solids 5, 365 (1971).

${ }^{7}$ M. V. Coleman and D. J. D. Thomas, Phys. Status Solidi 24, K111 (1967).

${ }^{8}$ R. Grigorovici and R. Mănăilă, Thin Solid Films 1, 343 (1968).

${ }^{9}$ R. Dandoloff, G. Döhler, and H. Bilz, J. Non-Cryst. Solids 35\&36, 537 (1980).

${ }^{10}$ S. Mader, J. Vac. Sci. Technol. 8, 247 (1971).

${ }^{11}$ Y. Saito, J. Cryst. Growth 47, 61 (1979).

${ }^{12}$ Ch. Janot and J. M. Dubois, J. Phys. F 18, 2303 (1988), Fig. 3.

13J. D. Joannopoulos and M. L. Cohen, Phys. Rev. B 7, 2644 (1973).

${ }^{14}$ M. Kléman and J. F. Sadoc, J. Phys. Lett. 40, L569 (1979)

${ }^{15}$ J. P. Sethna, Phys. Rev. Lett. 51, 2198 (1983).

${ }^{16}$ J. P. Sethna, Phys. Rev. B 31, 6278 (1985)

${ }^{17}$ D. R. Nelson, Phys. Rev. B 28, 5515 (1983).

${ }^{18}$ Y. Ishii, Acta Crystallogr. A 44, 987 (1988)

${ }^{19}$ D. Shechtman, I. Blech, D. Gratias, and J. W. Cahn, Phys. Rev. Lett. 53, 1951 (1984).

${ }^{20}$ For an introductory overview, see, e.g., D. R. Nelson, Scientific American, 255(2), 33 (1986).

${ }^{21}$ R. Mosseri and J. F. Sadoc, in The Structure of NonCrystalline Materials, 1982, edited by P. H. Gaskell, J. M. Parker, and E. A. Davis (Taylor and Francis, London, 1983), p. 137.

${ }^{22}$ A. L. Mackay, Nature 315, 636 (1985).

${ }^{23}$ C. L. Henley and V. Elser, Philos. Mag. B 53, L59 (1986).

${ }^{24}$ Z. Olami and S. Alexander, Phys. Rev. B 39, 1478 (1989).

${ }^{25}$ The high isotropy of the icosahedral symmetry is manifest, for example, in the fact that the low-order elasticity is truly isotropic or that the first nontrivial multipole moment in the bond-angle distribution function occurs at $l=6$.

${ }^{26} \mathrm{As}$ examples the elastic modulus, vibronic and electronic contributions to the specific heat, the magnetic behavior, and the electronic density of states are discussed on pp. 361-408 of Quasicrystalline Materials, Proceedings of the I.L.L./CODEST Workshop 1988, edited by Ch. Janot and J. M. Dubois (World Scientific, Singapore, 1988).

${ }^{27}$ Since the Penrose quasilattice contains $\left(6 \mathrm{P}_{3}, 2 \mathrm{O}_{1}\right)$ - and $\left(14 P_{1}, 6 O_{1}\right)$-vertex neighborhoods [C. L. Henley, Phys. Rev. B 34, 797 (1986), Table III], tetracoordination of such positions would require bonds pointing into the $P_{1}, P_{3}$, or $O_{1}$ corners. But this would result in at least sixfold coordinations. The existence of $\left(2 \mathrm{P}_{3}, 2 \mathrm{O}_{7}\right)$ and $\left(1 \mathrm{P}_{1}, 4 \mathrm{P}_{3}, 1 \mathrm{O}_{7}\right)$ vertices excludes decoration of $\mathrm{O}_{7}$ corners as well. Similarly, all edges must be left undecorated.

${ }^{28} \mathrm{~K}$. Miyazaki and I. Takada, [Topologie Structurale 4, 21 (1980)] missed noting this overlap and Mackay (Ref. 22) had to modify the stacking by the introduction of hexagons and square rings around the mentioned short diagonal.

${ }^{29}$ R. Grigorovici, in Amorphous and Liquid Semiconductors, edited by J. Tauc (Plenum, New York, 1974), p. 92; see also Ref. 6, p. 373; or Fig. 7 in Ref. 8.

${ }^{30} \mathrm{~A}$ rational approximant selects a finite section of the quasiperiodic pattern and repeats it periodicially (simple cubic).

${ }^{31} \mathrm{H}$. Richter and G. Breitling, Z. Naturforsch. Teil A 13, 988 (1958). 